\title{
Soft lithography meets self-organization: Some new developments in meso-patterning
}

\author{
RABIBRATA MUKHERJEE ${ }^{\dagger}$, ASHUTOSH SHARMA*, GANESH PATIL, \\ DANISH FARUQUI and PARTHO SARATHI GOOH PATTADER \\ Department of Chemical Engineering, Indian Institute of Technology, Kanpur 208 016, India \\ ${ }^{\dagger}$ Present address: Central Glass and Ceramic Research Institute, Kolkata 700 032, India
}

\begin{abstract}
This is a brief review of our recent and ongoing work on simple, rapid, room temperature, pressure-less and large area $\left(\sim \mathrm{cm}^{2}\right)$ imprinting techniques for high fidelity meso-patterning of different types of polymer films. Examples include soft solid polymer films and surfaces like cross-linked polydimethylsiloxane (PDMS) and polyacrylamide (PAA) based hydrogels, thermoplastics like polystyrene (PS), polymethylmethacrylate (PMMA) etc both on planar and curved surfaces. These techniques address two key issues in imprinting: (i) attainment of large area conformal contact with the stamp, especially on curved surfaces, and (ii) ease of stamp detachment without damage to the imprinted structures. The key element of the method is the use of thin and flexible patterned foils that readily and rapidly come into complete conformal contact with soft polymer surfaces because of adhesive interfacial interactions. The conformal contact is established at all length scales by bending of the foil at scales larger than the feature size, in conjunction with the spontaneous deformations of the film surface on the scale of the features. Complex two-dimensional patterns could also be formed even by using a simple one-dimensional master by multiple imprinting. The technique can be particularly useful for the bulk nano applications requiring routine fabrication of templates, for example, in the study of confined chemistry phenomena, nanofluidics, bio-MEMS, micro-imprinting, optical coatings and controlled dewetting.
\end{abstract}

Keywords. Self-organization; meso-patterning; visco-elastic film; imprinting; adhesion; soft lithography.

\section{Introduction}

Topographically patterned polymer films and surfaces, having sub-micron scale resolutions are important in a host of scientific and commercial applications like components of molecular electronics (Burn et al 1993), organic light emitting diodes (OLED) (Burroughes et al 1990), optoelectronic devices (Lee et al 2004), photodiodes (Lupton et al 2003), thin film transistors (TFT) (Salleo et al 2005), solar cells (Brabec et al 2001), biological sensors (Kros et al 2002), manipulation, tracking and high sensitivity detection of single molecules (Levene 2003), microfluidics (McDonalds et al 2000), patterned adhesives (Ghatak et al 2004), carbon-micro-electro-mechanical systems (C-MEMS) (Singh et al 2003), etc. In many applications, rapid prototyping of relatively large area $\left(\sim \mathrm{cm}^{2}\right)$, meso-patterned surfaces with simple features having sizes ranging from $200 \mathrm{~nm}$ to 100 microns are required. These include confined chemistry applications (Rhee et al 2005), lab on a chip devices (Chow et al 2002), surfaces for nano-biotechnology applications (Whitesides 2003) like single molecule enzymology (Rondelez et al 2005), proteomic or genomic arrays (Lee et al 2002), photodiode

\footnotetext{
*Author for correspondence (ashutos@iitk.ac.in)
}

arrays for sub retinal implant in bionic eye (Meyer 2002), patterned substrates for probing of cell behaviour (Ito 1999) including stem cell scaffolds for tissue engineering (Chin et al 2004), DNA stretching (Guo et al 2004) etc. In addition to all the above mentioned applications, most of which relate to patterned films on planar surfaces, topographically patterned curved surfaces are also important to a host of areas relating to optoelectronic, imaging and biomedical devices. For example, spherical patterned surfaces enable fabrication of distortion free charge coupled device (CCD) arrays with ultra-wide fields-of-view (Jacobs et al 2002). Fabrication of a stent, a specialty biomedical device implanted at the site of a blockage inside blood vessels, has a tube-like 3-D structure and requires efficient technology of patterning curved surface (JagurGrodzinski et al 2006). A robust, low cost patterning method for curved surfaces, particularly suitable for any random geometry including arbitrary curvature, is still not in place, as most of the existing lithography methods are suited for patterning planar surfaces.

Of all the available sub-micron, meso and nano scale surface patterning methods (Henzie et al 2006) which are broadly covered under the generic term 'lithography', photolithography is the most popular and widely used method because of its ability to rapidly produce high fidelity defect free surface features (Bratton et al 2006). How- 
ever, photolithography has some limitations like (i) it requires expensive instruments and facilities with high capital investment, (ii) the pattern resolution is limited by optical diffraction and obtaining sub $190 \mathrm{~nm}$ patterns require extensive technology upgradation, (iii) it is not suitable for patterning all types of polymers as only photosensitive resist materials (photoresists) can be directly patterned, (iv) it requires harsh processing conditions like exposure to UV-radiation and chemical etching, therefore, not suitable for patterning sensitive materials, for example, biological samples with living cells. Some of the limitations of photolithography, particularly the resolution limit, can be overcome by using the next generation lithography (NGL) methods like focused ion beam (FIB) milling (Gates et al 2005), electron beam writing (Fischer and Chou 1993), proton beam writing (Ansari et al 2004), immersion lithography, deep UV lithography and methods based on scanning probe microscopic techniques (SPM) (Stellacci 2006) that are capable of producing structures having linewidth down to $\sim 10 \mathrm{~nm}$. However, all of these methods also require costly instruments apart from the fact that they are serial and hence are not suited for rapid, large area patterning. Large area patterning applications require the initial mask or mould making by e-beam or FIB, followed by their replication using other soft lithography methods.

The search for suitable surface patterning methods, which can pattern different types of polymer surfaces over large areas without sophisticated tools and instruments have resulted in the 'soft lithography' group of methods (Xia and Whitesides 1998). Major soft lithography methods include nano imprint lithography (NIL) (Chou et al 1996) and its variants like room temperature imprint lithography (Khang et al 2001), low pressure imprint lithography (Khang et al 2004), hot embossing (Schift et al 2001), micro molding in capillaries (MIMIC) (Kim et al 1995), replica molding (REM) (Xia et al 1996), micro contact printing ( $\mu \mathrm{CP})$ (Kumar and Whitesides 1993), capillary force lithography (CFL) (Suh et al 2001), step and flash imprint lithography (SFIL) (Bailey et al 2000), lithographically induced self assembly (LISA) (Chou et al 1999) etc. In these methods, the minimum resolution is not diffraction limited and variety of polymers from thermoplastics to soft gels can be patterned (Di Bendetto et al 2005). Though the exact pattern transfer mechanism differs significantly from method to method, generically a polymer film or a layer is imprinted or molded with a rigid or a flexible stamp or a master pattern, which has the exact negative replica of the final desired pattern on it. The pattern transfer takes place either due to viscous or viscoelastic or plastic deformation and subsequent reorganization of the film material. The methods are parallel and hence intrinsically fast and can pattern over large areas. The final pattern formation is engendered by the confined self organization of the polymer in presence of the stamp.
However, even the soft lithography methods have some practical limitations. For example, the master pattern or the stamp necessary for most of the methods mentioned above is fabricated using some other lithography methods, directly or indirectly (in some cases a PDMS stamp is used, which is fabricated by molding on a rigid master). A critical parallel configuration between the stamp and the film needs to be maintained for high fidelity large area patterning. The patterns formed cannot be tuned in situ and for creating patterns of different size, shape and morphology, a new stamp is necessary at all times. In this review of our recent activities, we present methods in which some of the limitations of the existing techniques can be partially addressed. In particular, the motivation is to circumvent limitations on the following important aspects: (i) maintaining a parallel and conformal stamp configuration with respect to the surface, which is especially difficult for curved surfaces, (ii) maintaining uniform pressure and temperature over large areas, (iii) high pressure and temperature required for imprinting and (iv) detachment of stamp after imprinting without structural damage and loss of fidelity. In some of the proposed techniques here, top-down methods are combined with self-organization.

Self-organization or self-assembly is an omnipresent principle in nature, in which discrete components assemble together to form ordered structures. There are hundreds of examples of systems which are seen around us that are based on self assembly like formation of crystals, micelles, colloids, lipid membranes, self assembled monolayers etc (Whitesides and Grzybowski 2002). Self-assembly finds important applications in chemistry, physics, biology, nanoscience and technology, materials and manufacturing (Whitesides et al 1991). Though much of the studies in self-assembly have focused on molecular components, the domain of self assembly is not only limited to molecular and cellular levels but extends to many structural organizations on all scales, starting from molecules to galaxies which happen due to self assembly (Terfort et al 1997). Energy minimization remains the key motivation for self assembly at all scales. At the meso scale, the assembly can be engendered by underplay of various interaction forces like van der Waal's, electrostatic, ionic, gravitational, steric etc. Self assembly of larger components shows lot of promise and is extremely important in the emerging fields of nano-structured materials, bio-inspired templating, DNA computing and new developments in microelectronics. Self assembly or organization is also extremely important to many of the already mentioned soft lithography methods like micro molding in capillaries (MIMIC) (Kumar and Whitesides 1993; Kim et al 1995), capillary force lithography (CFL) (Suh et al 2001), lithographically induced self assembly (Chou et al 1999) etc. Some other routes of self organized nano and meso scale structure formations particularly involving polymers, which are finding increasing attention lately, include condensation induced 'breath' 
patterning (Widwaski et al 1994), flow and evaporation induced patterning (Deegan et al 1997), phase separation of block-copolymers (Lopez and Jaeger 2001), contact instability of thin elastic films (Ghatak et al 2000), dewetting of ultra thin polymer films (Reiter 1992, 1993; Sharma and Reiter 1996, 2002; Sharma and Khanna 1998; Sharma 2003), thin film instability due to thermal gradients (Harkema et al 2003) and imposed external force fields (like electric fields) (Schäffer et al 2000) etc. Interestingly, though 'bottom-up' or 'top-down' approaches are frequently mentioned while referring to engineering of small (nano or meso scale) structures, selforganization is strictly speaking, neither! Self-organization refers to the spontaneous shape-changes or shaping of material brought about by the inherent stability and dynamics of the system itself. The science of selforganization predominantly deals with the creation of the conducive or proper condition and environment at which components or objects can assemble or reorganize at the desired formation. The understanding of the effect of individual interaction forces on the self assembling system is central to the understanding of the process.

The other interesting yet not so useful aspect, which often hinders the technique based on self-organization as viable manufacturing method for bulk production of meso- and nano-scale structures, is lack of long range order of the structures, as in most cases they are isotropic and random. In contrast, 'top down' simple patterning methods based on soft lithography result in creation of regular and ordered structures. For example, nano imprint lithography (NIL) (Chou et al 1996), which is a rather simple and direct pattern transfer method, creates excellent ordered, high fidelity regular patterns. In contrast, dewetting of a thin polymer film which has much elegant science governing the evolution process, results in randomly arranged droplets.

Therefore, the important aspect to focus on self organized patterning is to align the self organized structures, so that long range order can be imposed on them. The concept is known as guided self organization, where lateral confinement in the form of a patterned mold or a stamp is used to align the structures. This paper, which is a mini review of some of the preliminary developments which has taken place in recent years in our group, presents some 'do-it-yourself' type methods for creation of large sub-micro and meso-scale structures, at low cost. It is our objective to develop the methods in such a way that (i) use of costly instruments and equipments is minimized, (ii) the methods developed should be generic to various types of polymers like thermoplastics, soft elastic polymeric films, soft gels, etc and not limited to certain specific class of materials only. For example, only photoresists can be directly patterned by photolithography, (iii) use of low cost commonly available items as much as possible. For example, even for implementing soft lithography methods, a patterned stamp is necessary, which in most cases have to be separately fabricated by some high end lithography methods like e-beam writing, FIB milling or photolithography and (iv) the method should be able to pattern films coated on non-planar and curved surfaces. Most of the existing lithography methods, including soft lithography methods are limited to patterning of films coated on planar surfaces.

In what follows, we show that flexible metal foils extracted from commercially available optical data storage discs like CD/DVD can be used as a readily available, low cost stamp for patterning, which easily can create linewidth down to $\sim 400 \mathrm{~nm}$. The use of a flexible stamp enables patterning of curved surfaces, as a patterned foil, which has no structural rigidity bends along the contours of the film to make full contact. Additionally, foils from CDs can be pre-patterned by data storage in these discs (for example, burning a CD) before stripping the foil. We also use patterned flexible stamps of water soluble polymer polyvinylalcohol (PVA) in order to overcome the problems of mold rupture and mold removal that frequently occur when thin foils (like cross linked PDMS, fluropolymer etc) are used as stamps for patterning curved surfaces.

In this paper, we thus present a surface patterning method, that offers low cost, large area sub-micron patterning capability for soft films and surfaces. The method is thus particularly suitable for laboratory scale, bulk nano applications and for researchers who are non-experts in the field of lithography, but routinely require patterned surfaces as templates for their experiments. The proposed method will be particularly useful in many applications in which rapid prototyping of relatively large $\left(\sim \mathrm{cm}^{2}\right)$ mesopatterned polymer surfaces with simple features having sizes ranging from $200 \mathrm{~nm}-10$ microns are required in large quantities. Applications involving structures on these length scales include areas like carbon-MEMS and microbattery, controlled dewetting on patterned surfaces (Mukherjee et al 2007a) and surfaces for nano-biotechnology applications etc. Also, an easy availability of patterned surfaces on these scales can enable in situ investigations of interesting phenomena and their dynamics with optical microscopy.

\section{Materials and methods}

In this paper, we cover the patterning of three different classes of polymers, viz. (i) thermoplastic polymers, like polystyrene, which behave like liquids above glass transition temperature. The polymers can also be made liquidlike by exposing them to their solvent vapour, (ii) soft cross-linked elastic films, like cross-linked PDMS and (iii) soft gels, like polyacrylamide based hydrogels. While the apparent pattern transfer methods are nearly the same in all the cases, the conditions of patterning are different for the different types of polymers. In case of thermoplastic 
polymer films, solvent vapour exposure was employed to make the film liquid-like and subsequent transfer was by viscous deformation of the softened film. In contrast, the pattern transfer was achieved directly in the solid state in case of the elastic films and hydrogels, engendered by an attractive adhesive interaction between the patterned foil and the film surface. Patterning of both elastic as well as liquid films is also demonstrated in this paper. The key ingredients for the work, which are listed in this section, are (i) the patterned flexible stamps or masters (CD/DVD foils, PVA foils), (ii) the film materials (polystyrene, cross-linked PDMS, PAA hydrogels), (iii) the flat and patterned substrates, (iv) film preparation methods on flat and curved substrates and (v) patterning methods.

\subsection{Patterned flexible stamps: Optical data discs}

The protective aluminum coating of optical data storage discs like compact discs (CD) or digital video discs (DVD) have been used as thin flexible masters for imprinting. An optical data storage disc is typically made of a polymer (polycarbonate) disc, on which a single spiral track is drilled. The typical width and depth of each line in the spiral track are $800 \mathrm{~nm}$ and $130 \mathrm{~nm}$, respectively and the periodicity of the tracks is $\sim 1.5 \mu \mathrm{m}$, as shown in the AFM scan in figure 1(a). For a DVD foil, the stripe width is $\sim 400 \mathrm{~nm}$, periodicity, $\sim 800 \mathrm{~nm}$ and height, $\sim 70 \mathrm{~nm}$ (figure 1(b)). The diameter of a typical CD or a DVD is much larger than the separation between two lines and hence under an optical microscope the lines appear parallel with nearly infinite radii of curvatures (Emmelius et al 1989). The aluminum coating on the polycarbonate membrane also has the structural patterns that appear as parallel lines under an optical microscope. The polycarbonate disk and the aluminum foil could be separated and we have used the patterned aluminum foil as the stamp in some of our experiments. It should be noted that, polycarbonate part as well as the foil portion of virgin or blank CDs have been used before for micro contact printing for generating patterns using coloured ink (Chowdhury et al
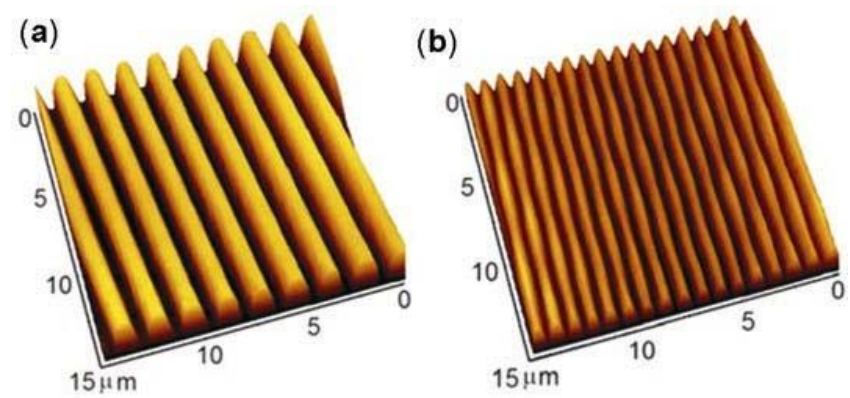

Figure 1. AFM scans of the peeled patterned foils of (a) a compact disc (CD) (stripe periodicity, $1.5 \mu \mathrm{m}$; stripe width, $750 \mathrm{~nm}$; stripe height, $130 \mathrm{~nm}$ ) and (b) a DVD (stripe periodicity, $800 \mathrm{~nm}$, stripe width, $\sim 400 \mathrm{~nm}$; stripe height, $\sim 70 \mathrm{~nm}$ ).
2001; Mukherjee et al 2007b). The patterned side of the foil, prior to being used in the imprinting process, is washed with methanol to remove any impurity and particularly the pigments, which are present in case of a DVD.

\subsection{Patterned flexible stamps: Patterned PVA foils}

For making the patterned PVA foils, the polycarbonate parts of commercially available optical discs like CD and DVDs were used as the original patterned stamp. The patterned aluminum part was peeled off as discussed already, and the polycarbonate parts were cut into small pieces. The pieces were washed with methanol to remove any impurity, particularly the pigments and subsequently dried in a stream of dry nitrogen. Other masters like photolithographically patterned silicon surfaces or any other type of patterns were also used for fabrication of PVA molds. PVA powder $\left(M_{\mathrm{W}} 1,25,000\right.$; SD-fine Chemicals, India) was dissolved in deionized millipore water (resistivity, $18.4 \mathrm{M} \Omega$ ) to make $\mathrm{a} \sim 12 \% \mathrm{w} / \mathrm{w}$ aqueous solution. The solution was stirred continuously with a magnetic stirrer for sometime. The resulting viscous solution was spin coated or poured onto the patterned polycarbonate pieces to form PVA films and subsequently air dried for $\sim 6 \mathrm{~h}$ to remove all the solvent (water). The air dried PVA films were further annealed in low vacuum for $18 \mathrm{~h}$ to remove any residual solvent (water). The annealed films were subsequently peeled off from the original patterned stamps. Figure 2(a) shows the digicam image of a $\sim 70 \mu \mathrm{m}$ thick, $40 \times 10 \mathrm{~mm}$ piece of a peeled and dried PVA foil, which was created by using a CD master. The existence of the patterns can be visually ascertained from the optical diffraction seen on the foil. A detailed investigation of the foil topography was done by using an AFM, which is shown in figure 2(b). The AFM image shows that the height of the stripes on the PVA foil is $\sim 130 \mathrm{~nm}$, periodicity, $\sim 1.5 \mu \mathrm{m}$ and stripe width, $\sim 750 \mathrm{~nm}$, signifying that it is a perfect negative replica of the mold used (the polycarbonate part of a $\mathrm{CD}$ ).

\subsection{Film materials}

2.3a Preparation of thermoplastic thin films: Various types of polymer films like polystyrene (PS) $\left(M_{\mathrm{W}}=\right.$ 280,000; Aldrich, USA), polymethylmethacrylate (PMMA) $\left(M_{\mathrm{W}}=196,000\right.$; Aldrich, USA), polycarbonate (PC) $\left(M_{\mathrm{W}}=\right.$ 96,000; SD-fine Chemicals, India) and polysulfone (PSf) $\left(M_{\mathrm{W}}=72,000\right.$; SD-fine Chemical, India) were used as the model systems for patterning of liquid films. The solvent used was HPLC grade Toluene (e-Mark, India).

2.3b Preparation of cross-linked PDMS films: For making soft elastic films, cross-linked polydimethylsiloxane (PDMS) films, having thickness in the range $300 \mathrm{~nm}-10 \mu \mathrm{m}$ and shear moduli in the range $\sim 0 \cdot 1-1 \mathrm{MPa}$ (as measured 

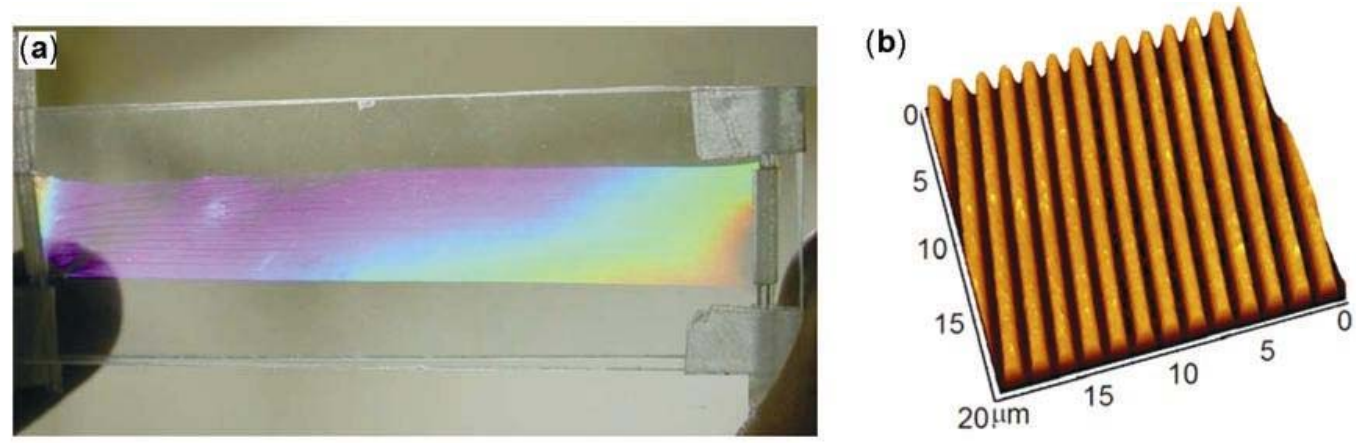

Figure 2. (a) Digicam image of a $10 \times 40 \mathrm{~mm}$ patterned flexible PVA foil and (b) AFM scan of the foil shown in (a), showing that the stripe periodicity is $1.5 \mu \mathrm{m}$, stripe width, $750 \mathrm{~nm}$ and stripe height, $130 \mathrm{~nm}$.

by a rheometer, Bohlin Instruments), were cast on thoroughly cleaned quartz substrates (typical size, $15 \times 15 \mathrm{~mm}$ ) by spin coating Sylgard-184 with 5\% cross-linker (a twopart PDMS elastomer, Dow Chemicals) from its solution in HPLC grade $n$-heptane. The film thickness was controlled by varying the dilution of the polymer in solvent, the RPM and the duration of the spinning process. For non-planar substrates, dip coating was employed. The films were subsequently cured at $120^{\circ} \mathrm{C}$ for $12 \mathrm{~h}$. In both the cases, involving PS films and Sylgard 184 films on flat substrates, film thickness was measured by an imaging ellipsometer (Nanofilm GmbH, Germany) for the thinner films (up to $800 \mathrm{~nm}$ ) and a surface profilometer for thicker films.

2.3c Preparation of polyacrylamide (PAA) hydrogel films: The polyacrylamide (PAA) based hydrogel was prepared by the polymerization of acrylamide and $N, N$ methylenebis(acrylamide) (MBAA, cross-linking agent) in an aqueous phase. A solution of acrylamide $(12 \% \mathrm{w} / \mathrm{v})$ and MBAA $(5 \% \mathrm{w} / \mathrm{v})$ in water was prepared, to which $N, N, N, N$-tetramethylethylenediamine (TEMED) $(1.5 \% \mathrm{w} / \mathrm{v})$ was added as a catalyst. The polymerization reaction was initiated by the addition of ammonium persulfate $(0.5 \%$ w/v). The reaction was allowed to proceed at room temperature, and the gelation time was about $5 \mathrm{~min}$. In order to prepare the hydrogel films, $\sim 200 \mu$ l of the reaction mixture was cast on a cleaned glass slide $(20 \times 20 \mathrm{~mm})$ and allowed to spread on it to form a film. Within $5 \mathrm{~min}$ of casting the reaction mixture polymerized into gel on the glass slides. The initial water content of the soft gels was $\sim 60-70 \%$ at the time of casting.

\subsection{Substrates}

2.4a Flat substrates: Cleaned silicon wafer pieces were used as substrates for experiments with PS as well as soft PAA hydrogels. Cleaned quartz pieces were used for experiments with cross-linked Sylgard 184 films. Both the types of substrates were thoroughly cleaned before coating. The substrates were first soaked and sonicated in detergent solution for $30 \mathrm{~min}$, followed by boiling in organic solvents like trichloro ethylene (TCE), acetone and methanol, fifteen min each. The substrates were then washed with water and soaked in heated $5: 1: 1$ solution ( 5 parts by volume water, 1 part by volume $\mathrm{H}_{2} \mathrm{O}_{2}$ and 1 part by volume $\mathrm{NH}_{4} \mathrm{OH}$ ) for 30 min followed by soaking in chromage solution (potassium dichromate in conc. $\mathrm{H}_{2} \mathrm{SO}_{4}$ ) for another $30 \mathrm{~min}$. The substrates were washed with deionized millipore water (resistivity: $18.4 \mathrm{M} \Omega$ ) and dried in a stream of nitrogen, just prior to coating.

2.4b Non-planar substrates: Curved surfaces of different materials having various geometries and sizes were used for the experiments. Some typical items used as substrates included the outside of glass test tubes, having diameter in the range $\sim 1 \mathrm{~mm}-\sim 1 \mathrm{~cm}$ and length, $\sim 2-4 \mathrm{~cm}$, polished steel tubes having similar diameters, glass spheres having typical diameter in the range $\sim 5-20 \mathrm{~mm}$, shallow glass dishes etc. Before coating, the substrates were cleaned to remove any contaminant or impurity on the surface, following the protocol described in the preceding section. For the metallic substrates, boiling in $5: 1: 1$ solution and soaking in chromage solution were not done.

\subsection{Film preparation methods}

2.5a Coating of polymer films on flat substrates: Spin coating was adopted for coating the films on flat substrates. The thicknesses of the films were controlled mainly by varying the concentration of the solution, spin speed and also to a lesser extent, by the duration of spinning.

2.5b Coating of polymer films on curved substrates: Automatic dip coating was done to coat the films, by fixing the substrates onto the dipper unit of a Lang- 


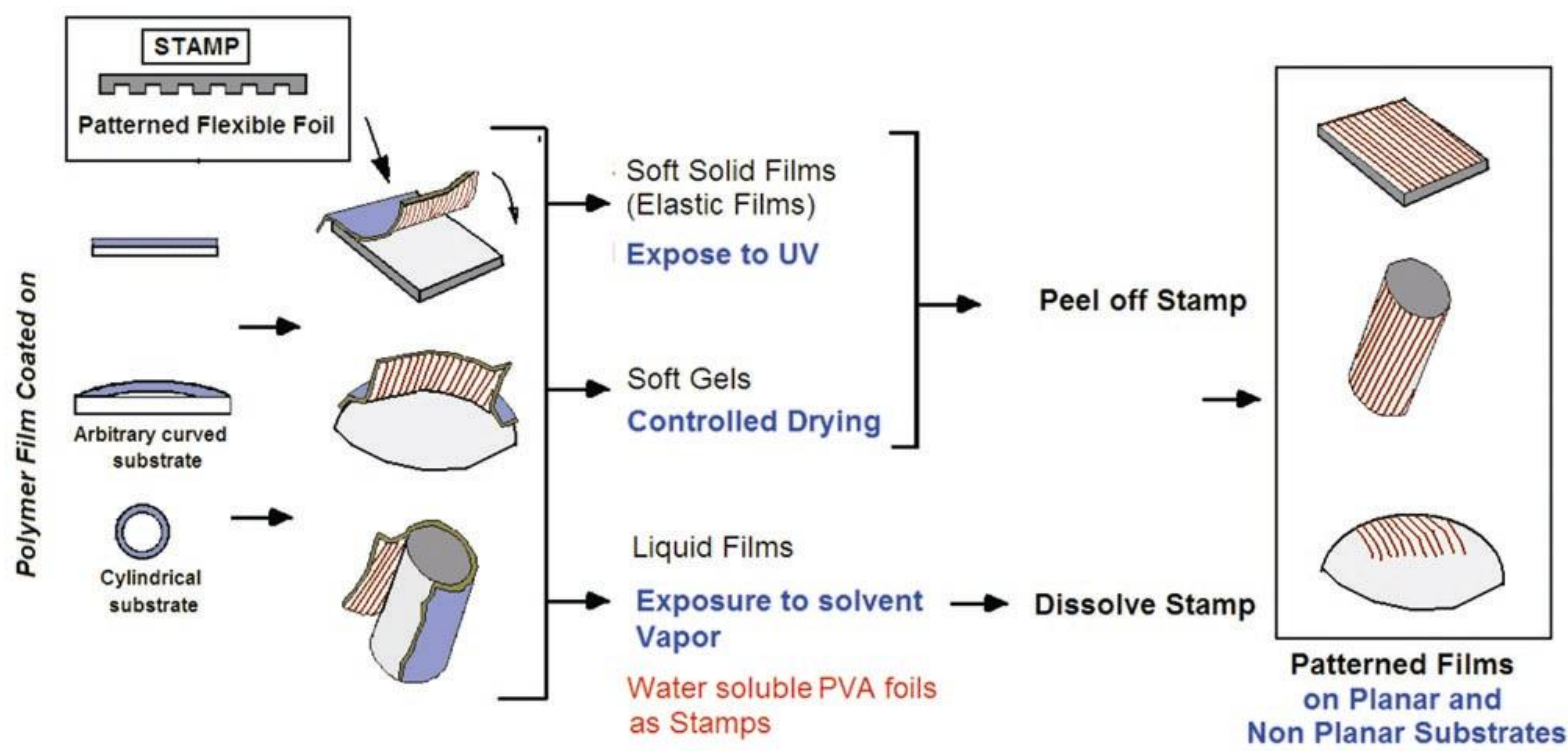

Figure 3. Schematic of different soft imprinting methods using flexible patterned foils as stamps, reported in the paper.

muir Blodget Trough (NIMA, UK, Model: 1212D2). The dipping speed was kept high, at $\sim 12 \mathrm{~mm}$ per min and the number of dips was varied between 6 and 30 , which resulted in films having thicknesses in the range $750 \mathrm{~nm}-30 \mu \mathrm{m}$. Larger tubes and substrates, which could not be fitted to the dipper, were coated manually. The film thicknesses were measured by measuring the weight difference and dividing it by the area over which the film covered the substrates. Such a method of thickness measurement, it must be emphasized, results in a very gross approximation, which had to be used in absence of any other possible method for assessing the film thicknesses on curved substrates. For tubes (particularly when test tubes were used), the closed ends were chosen for coating, as this ensured only the outer surface and not the inner surface was coated during dip coating.

\subsection{Patterning methods}

2.6a Adhesion assisted patterning of elastic films: The cleaned patterned foil pieces, with the patterned side up, were placed carefully on glass slides. The Sylgard or the hydrogel films were then gently placed on the patterned foils (as shown in figure 3 ). As the slides were placed on the foil pieces, the only compressive force the soft films were subjected to was the weight of the quartz slides, which was $\sim 1 \mathrm{~g}$. This corresponds to a pressure of $\sim 4.5 \times$ $10^{-3} \mathrm{MPa}$. However, similar to our earlier observations in experiments with a rigid stamp (Gonuguntla et al 2006a), there is a strong attractive interaction between the stamp and the film, once the two are in very close proximity to each other. This adhesive interaction primarily originates from the attractive van der Waal's forces acting between the two surfaces. The patterned foil, which does not have adequate structural rigidity, succumbs to this attractive interaction and comes in full conformal contact with the film. Once in full contact, the weight of the quartz or glass slides are enough to deform the top surface of the soft films at a length scale corresponding to each structure on the stamp. This deformation is purely elastic not due to the bending of the flexible foil, which facilitates the film and the stamp are at conformal contact, ensuring the formation of a perfect negative replica over large areas. This conformal bending of the foil is utilized while patterning non-planar curved surfaces, where the film is dip coated on a curved surface (cylindrical, spherical or arbitrarily curved). The physics of the pattern transfer remains the same irrespective of the surface geometry. Interestingly, PVA foils were used, instead of the patterned aluminum foils for patterning Sylgard films on curved surfaces. The use of PVA foils ensured that the UV irradiation reached the film-foil interface leading to the necessary surface hardening.

The imprinting on the surface of the film is engendered largely by the elastic deformation in case of cross-linked PDMS layer, as evidenced by a rapid disappearance of the imprint upon removal of the foils. The patterns could, however, be made permanent by a UV-ozone treatment for $20 \mathrm{~min}$. UV irradiation at $185 \mathrm{~nm}$ produces ozone from atmospheric oxygen, which further gets dissociated to atomic oxygen at $254 \mathrm{~nm}$ irradiation (Ouyang et al 2000). This atomic species reacts with the siloxane group, releasing volatile products and leaving behind a stiff surface layer consisting of oxides of silicon. The higher modulus of the surface layer prevents the relaxation of the film and preserves the relief patterns imprinted on the 
soft surface by the foil. On the hydrogel films, patterns were made permanent by complete drying of the film, while in contact with the stamp. By keeping the film in complete contact with the stamp during drying prevented lateral shrinkage of the patterns and resulted in perfect negative replica of the stamp pattern on the hydrogel film surface.

2.6b Solvent vapour assisted imprinting of liquid polymer films: The schematic of the solvent vapour assisted imprinting process using flexible PVA stamps is also shown in figure 3 . Briefly, the coated polymer films were placed inside a solvent vapour chamber (a covered glass desiccator). Before placing the films, the chamber was pre-saturated with solvent vapour (toluene vapour for PS and PMMA and chloroform vapour for PSf and PC) for about $2 \mathrm{~h}$. In order to prevent solvent condensation within the chamber, the experiment was carried out at room temperature. The films were allowed to swell in the controlled solvent atmosphere and once swelled, were then wrapped conformally with the patterned PVA foil. A good adherence between the PVA foil and the soft swollen polymer film was ensured by gentle finger pressure, particularly to remove any entrapped air bubbles. The film, with wrapped stamp was further exposed to solvent vapour in the chamber for about $90 \mathrm{~min}$, which was found to be sufficient for pattern transfer. During this period, the reduced viscosity, swollen polymer first rises along the walls of the stamp due to capillary forces, until the advancing meniscus reaches the ceiling (the bottom of the valleys on the stamp surface). Subsequently, complete 'mold filling' takes place, where the entire space under each valley of the stamp is filled up by the liquid polymer, leading to the formation of a perfect negative replica of the stamp on the film surface. Once the replica forms, the PVA mold or stamp is washed and dissolved in a stream of deionized water, exposing the patterned film surfaces. The duration of wash varied between $5 \mathrm{~min}$ and $15 \mathrm{~min}$, depending on the thickness of the PVA stamp. The washed, patterned films were investigated under optical microscope as well as scanned with an atomic force microscope (AFM) to ensure that all the mold material (PVA) has been removed properly and also to investigate the quality and fidelity of the transferred patterns.

\section{Results and discussion}

\subsection{Adhesion assisted patterning of elastic films}

3.1a Imprinting on flat substrates: Figures 4(a) and (b) show the patterns obtained on $3.1 \mu \mathrm{m}$ and $2.2 \mu \mathrm{m}$ thick cross-linked PDMS films using CD and DVD foils as stamps, respectively. The imprinted patterns, obtained by elastic deformation of the soft PDMS film, directly in the solid state, have high fidelity with sharp edges, as can be seen in the 3-D rendered AFM images, shown in the insets al and bl of figure 4 . The periodicity, linewidth and height of the stripes reflected the fact that the imprinted patterns were indeed perfect negative replicas of the patterned foils used for imprinting. Using this technique, perfect negative replicas of the stamps on films of various thicknesses could be achieved. Unlike the emerging NIL based methods, the proposed method differs in two key aspects - a flexible foil rather than a rigid stamp is used for facilitating a pressureless uniform and conformal contact with the film. Importantly, the conformal contact of flexible foil is facilitated by adhesive forces rather than by the application of external pressure. Finally, the most important aspect is that the pattern transfer of imprinting takes place directly in the solid state.

3.1b Complex 2-D patterns by multiple imprinting: Complex 2-D structures from a simple 1-D mold could also be generated by an intermediate withdrawal, rotation and reapplication of the stamp, during UV exposure. The film in the presence of the mold was first partially hardened by the UV irradiation for a short duration $(\sim 5 \mathrm{~min})$ to prevent its immediate relaxation. The mold was then withdrawn, rotated and brought back into contact with the stamp at an angle to the initial patterns and subsequently cured with UV-ozone for another $25 \mathrm{~min}$. Such 2-D structures created by this method are shown in figures 5(a) and (b), respectively. To create the pattern shown in figure 5(a), a DVD stamp was imprinted twice on a $2 \cdot 2 \mu \mathrm{m}$ film, with the second imprint at right angles to the direction of the first imprint. The arched cross sections of the raised parts of the films resemble the curved profile of the lines on the original DVD mold. For the pattern shown in figure 5(b), a CD foil was imprinted twice on a $1.9 \mu \mathrm{m}$ thick cross-linked PDMS film. However, in this case, the direction of second imprint was at $\sim 45^{\circ}$ to that of the first imprint.

An important advantage of the proposed method is its large area patterning capability as well as ability to pattern films coated on curved or non-planar surfaces. Both these advantages could be simultaneously achieved because of using a thin patterned foil as the stamp, instead of a rigid stamp. Using the method described, we could reproducibly pattern areas as large as $2 \times 2 \mathrm{~cm}$ on a planar surface without using any step and repeat procedure (Ruchhoeft et al 1999) or roller nano-imprint machine (Tan et al 1998).

3.1c Adhesion assisted patterning of elastic films on curved substrates: The digicam image of figure 6(a) shows the patterns created by this method on a circular glass tube coated with cross-linked PDMS film. For patterning on a curved surface, we used patterned polyvinyl alcohol (PVA) foils as stamps (Schaper 2003) as the material is transparent to UV light, thereby exposing the filmstamp interface to UV light for making the patterns per- 

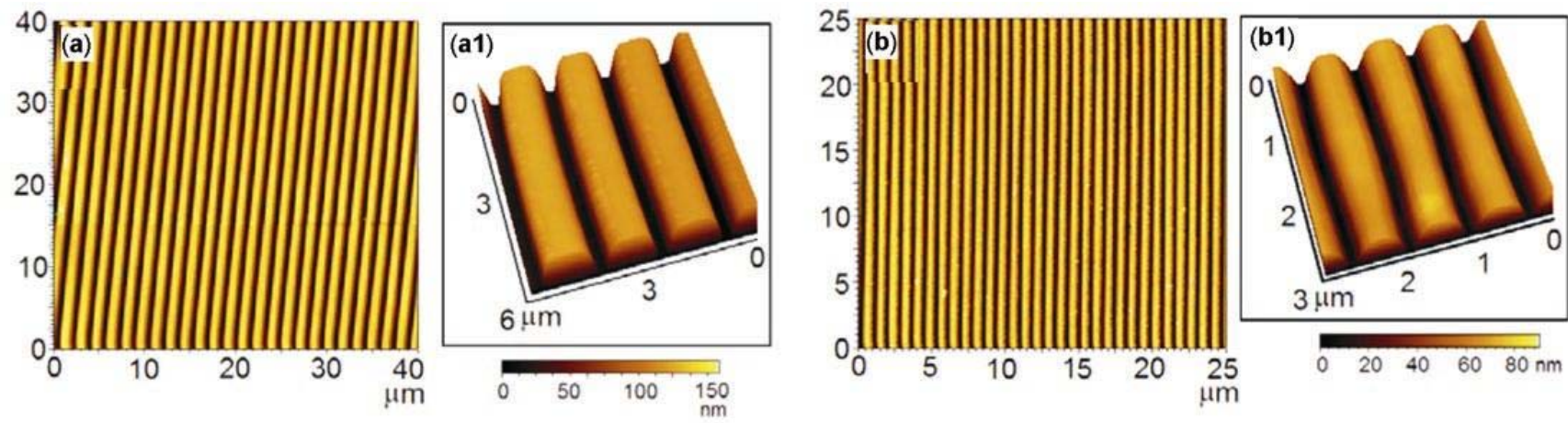

Figure 4. Typical AFM images of negative replicas of foil patterns on cross-linked PDMS films. Stripe patterns obtained using (a) a CD foil (film thickness, $3 \cdot 1 \mu \mathrm{m}$ ), and (b) a DVD foil (film thickness, $2 \cdot 2 \mu \mathrm{m}$ ). Heights of the polymer patterns match with the height of foil patterns. Insets are 3-D AFM images, which clearly show the high fidelity of the patterns in case of using CD foils and arch like cross-section while using DVD foils.
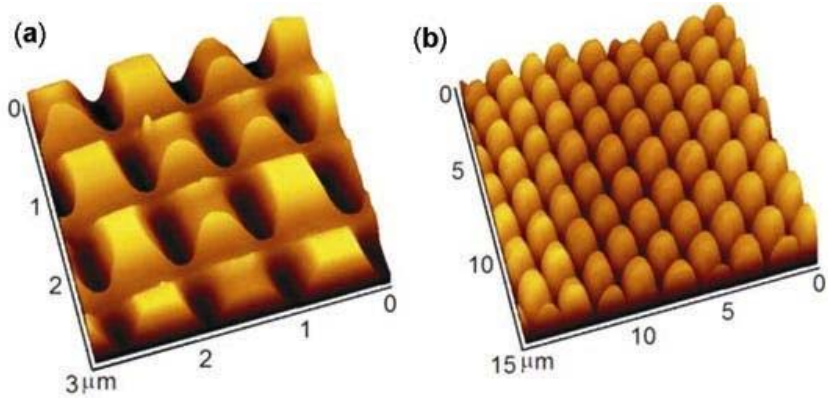

Figure 5. AFM images of two-dimensional cross patterns obtained by multiple imprinting. (a) $350 \times 350 \mathrm{~nm}$, pillars obtained by multiple imprinting using a DVD stamp. The second imprint was at right angles to the direction of the first imprint and the film thickness was $\sim 2.3 \mu \mathrm{m}$ and (b) rhombic columns obtained by imprinting the PDMS film twice with a CD stamp, by making the second imprint at an angle of $45^{\circ}$ relative to the first. The film thickness was $\sim 1.9 \mu \mathrm{m}$.

manent. In case of patterning of curved surfaces too, the pattern transfer takes place directly in the solid phase, in contrast to existing reported methods where the liquid is heated beyond its glass transition temperature and the patterning takes place in liquid state. Figure 6(b) shows the patterns imprinted on a glass test tube, having $14 \mathrm{~mm}$ outer diameter, coated with a $5.76 \mu \mathrm{m}$ thick cross-linked PDMS film and imprinted with a PVA foil created from a CD mold. Figure 6(c) shows 2-D array of square pillars created by multiple imprinting, using PVA foils created using $\mathrm{CD}$ molds twice, with the direction of the imprints at right angles to each other. In this particular case, the film thickness was $\sim 4.3 \mu \mathrm{m}$ and it was coated on a circular tube having a diameter of $16 \mathrm{~mm}$. For the first imprinting, a $\mathrm{CD}$ foil was used while another $\mathrm{CD}$ foil was used for the second imprinting. A small portion of the tube was cut to enable AFM imaging.

\subsection{Adhesive imprinting of hydrogel films}

Hydrogels, on the other hand, are a special class of soft materials which have wide chemical as well as biological applications for their inherent bio-compatibility and ability to respond to host of external stimuli like variation in $\mathrm{pH}$ (Tanaka et al 1980), ionic strength (Eichenbaum et al 1998), temperature (Hu et al 1995), electric field (Hu et al 1998) etc. Micropatterned hydrogel films and surfaces find important applications in areas like cell adhesion and growth (McFarland et al 2000), cell biology and tissue engineering (Kane et al 1999), drug delivery (He et al 2004), flow actuators in microfluidics (Beebe et al 2000), etc. Patterning methods generally adopted for hydrogel surfaces are by photo-polymerization of liquid monomers/precursors, by micromolding in capillaries (Unger et al 2000) or by transfer of pattern from a photoresist coating on the gel, patterned by conventional optical lithographic techniques followed by etching (Lei et al 2004) or by soft lithographic techniques (Di Bendetto et al 2005). Gentle patterning of hydrogels, especially with embedded cells, without significant structural alterations and damage is a rather challenging task and the usual photolithographic procedures involving radiation curing and etching are not always suitable.

Figures 7(a) and (b) show the patterns obtained on $\sim 3.5 \mu \mathrm{m}$ and $6.1 \mu \mathrm{m}$ thick hydrogel (PAA) films using a CD and the DVD foil as stamps, respectively. The width, height and periodicity of the stripes obtained using a CD mold were $792 \pm 12 \mathrm{~nm}, 115 \pm 4 \mathrm{~nm}$ and $1.42 \pm$ $0.085 \mu \mathrm{m}$, respectively. The same parameters obtained using a DVD were $281 \pm 12 \mathrm{~nm}, 64 \pm 2 \cdot 3 \mathrm{~nm}$ and $782 \pm$ $16 \mathrm{~nm}$, respectively. The flexible stamp results in close conformal contact with hydrogel film surface, thereby ensuring the formation of a perfect negative replica of the stamp pattern, unlike a variety of intermediate structures observed by others (Gonuguntla et al 2006b), while pattern- 

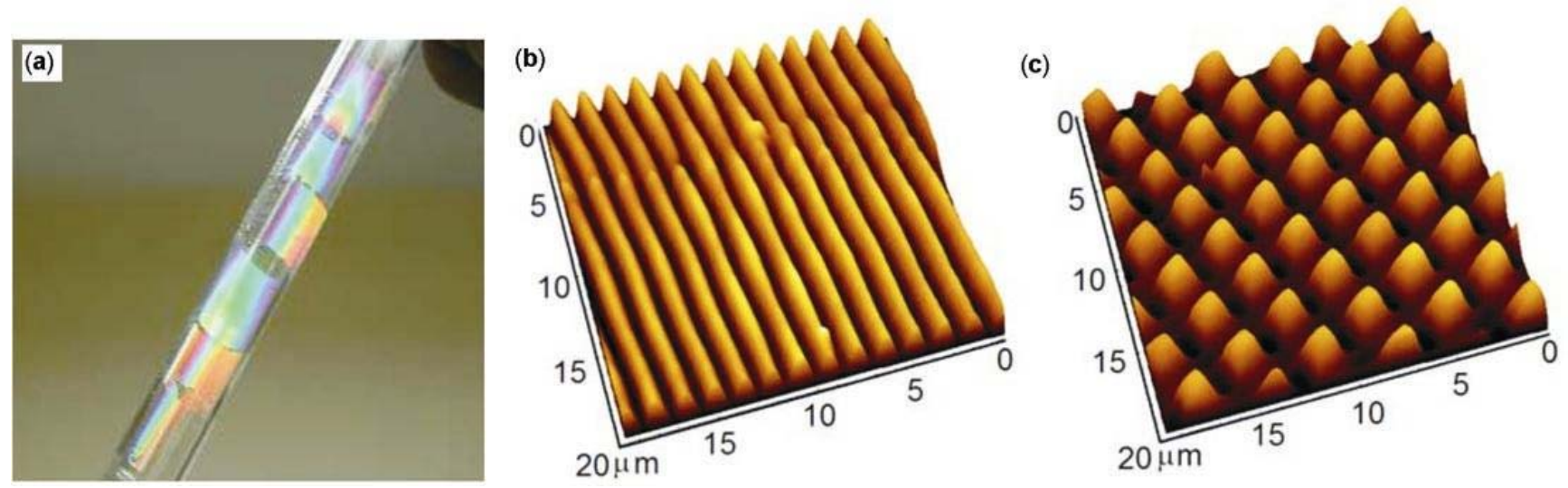

Figure 6. (a) Digicam image showing large area pattern on a PDMS film, coated on a cylindrical glass surface. The length of the patterned area is $\sim 40 \mathrm{~mm}$. (b and $\mathbf{c}$ ) AFM images of patterns on cross-linked PDMS films, coated on curved substrates, and using stripe patterned PVA foils having a periodicity of $\sim 1.5 \mu \mathrm{m}$, (b) 1 -D stripe patterns obtained on a $5.76 \mu \mathrm{m}$ thick film by single imprinting and (c) 2 -D cross patterns on a $4.3 \mu \mathrm{m}$ thick film, by multiple imprinting.

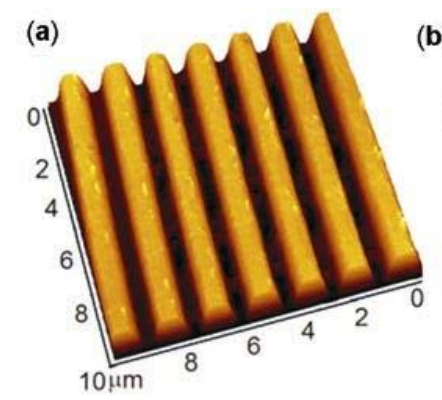

(b)

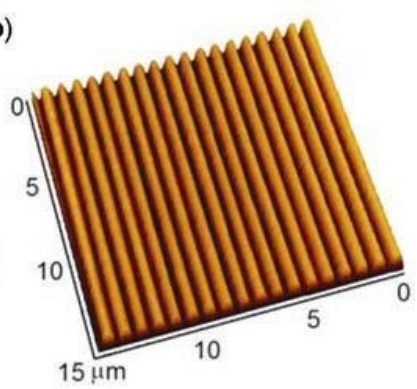

Figure 7. AFM images of negative replicas of foil patterns on hydrogel (PAA) films obtained by adhesion assisted imprint lithography. (a) Stamp used is a CD foil and (b) a DVD foil. The film thickness were $3.5 \mu \mathrm{m}$ and $6.1 \mu \mathrm{m}$, respectively

ing hydrogel thin films with patterned rigid stamps. The critical stage while patterning hydrogel films by this method was the gelation time, which was a function of the initial volume of the reaction mixture added to cast the film. The other critical stage was the drying of the film while in contact with the stamp. For thicker hydrogel films, the drying was associated with buckling of the film, which resulted in peeling off of the film from the substrate (glass slide). Thinner films $(<1 \mu \mathrm{m})$, on the other hand, resulted in breaking up of the film into isolated patches while drying.

\subsection{Solvent vapour assisted imprinting of thin liquid films}

3.3a Imprinting films coated on planar substrates: Figure 8(a) shows the imprinted patterns on a $168 \mathrm{~nm}$ thick PMMA film, coated on a silicon wafer substrate and imprinted with a PVA foil, having a periodicity of $\sim 1.5 \mu \mathrm{m}$. The film was first kept inside a solvent vapour chamber for $60 \mathrm{~min}$, before imprinting. Once the film was imprinted with the PVA foil, it was again kept inside the solvent vapour chamber for about $90 \mathrm{~min}$. The post-exposure time varied between 60 min to $\sim 3 \mathrm{~h}$, depending on the variation in the film thickness.

The pre-exposure allows the solvent molecules to penetrate into the polymer matrix thereby swelling the film and making it soft enough for room temperature imprinting. In contrast to adhesion assisted imprinting of elastic films and soft hydrogels, where the pattern transfer takes place in the solid state, here the patterning is engendered by the deformation and subsequent reorganization of a soft, viscous film layer. The key parameter in obtaining a perfect imprinting by this method is the optimized exposure times, both before and after imprinting. For example, if the pre-exposure time is too less, it will result in low fidelity imprints. Multiple imprints, in this case too resulted in complex 2-D patterns, details of which will be discussed later, while reporting the solvent vapour assisted pattern formation of films coated on curved substrates.

A more interesting scenario emerges when the thickness of the polymer film is gradually reduced. Particularly, for film thicknesses $<100 \mathrm{~nm}$, it was no longer possible to create negative replicas of the stamps. Instead, the film dewetted under the mold and formed aligned array of dewetted droplets as shown in figure 8(b) for a $59 \mathrm{~nm}$ thick PMMA film. For thin films, of particular importance, was the pre-exposure time as a long preexposure rendered the film completely dewetted, even before it could be imprinted with the patterned foil. However, when a high molecular weight polymer film was used, the dewetting could be successfully suppressed by reducing the exposure times (exposure time before and after imprinting). Confined dewetting under molds has been reported in the literature earlier and therefore, the details are not provided here. It is important to note that depending on the film thickness, mold geometry and exposure times, 
host of interesting structures, and not only a mere negative replica of the stamp could be created in solvent vapour assisted imprinting method. The PVA foil, which was used as the stamp was simply washed and dissolved with deionized water to expose the imprinted patterns without damaging them. The use of a flexible PVA foil thus reduces the chances of mold rupture, frequently encountered with various other types of thin flexible patterned stamps (like PDMS, fluropolymer etc) while peeling after pattern transfer. Small pieces of the ruptured stamp remains stuck to the patterned surface, making it unsuitable for further processing or use. In contrast, a PVA foil is neatly washed off, leaving no marks on the patterned surface.

3.3b Solvent vapour assisted imprinting of films coated on curved substrates: Figure 9(a) shows digicam photographs of a polymer film coated on non-planar substrates with spherical and cylindrical geometries, over large areas, using the proposed method. The existence of the diffraction colours visually signifies the imprinting of the polymer films. The presence of multiple colours in the diffraction pattern is due to the curvature of the substrates themselves. The details of the imprinted patterns on the curved surfaces were investigated using both optical microscopes as well as by scanning with an AFM. The images of 1-D negative replica (stripe patterns) are not shown as they are similar in appearance to the image shown in figure $8(\mathrm{a})$.

Complex 2-D structures created from simple 1-D stripe patterned PVA stamp could also be created following the proposed method by multiple imprinting with and intermediate stamp removal and reapplication of a new stamp, perpendicular (or can be aligned at any angle) to the direction of the first imprinted stripes. Figures 9(b) and (c) show the 2-D ordered patterns that were created on the surface of a $7.2 \mu \mathrm{m}$ and $4.8 \mu \mathrm{m}$ thick PS films, respecti-
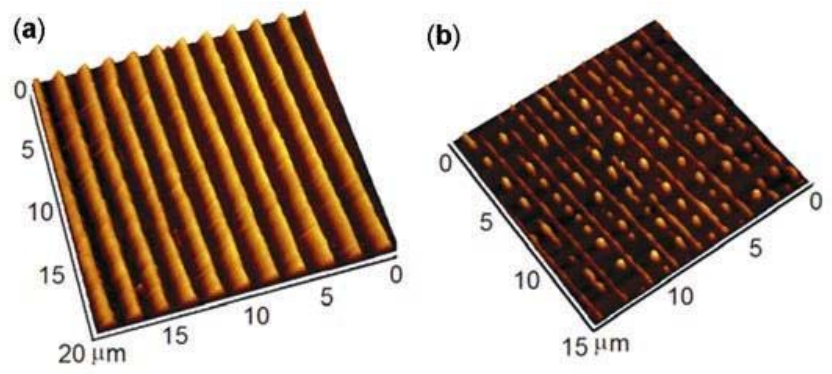

Figure 8. AFM images of patterns obtained on thin PMMA films coated on flat silicon wafer substrate by solvent vapour assisted imprinting at room temperature using flexible patterned PVA foils. (a) A $168 \mathrm{~nm}$ thick PMMA film imprinted to form a perfect negative replica of the stamp and (b) imprinting of a 59 nm thick PMMA film under a patterned PVA stamp resulted in confined dewetting of the film, with formation of aligned dewetted structures. vely, using two PVA stamps, both having stripe patterns. For the pattern shown in figure 9(b), the stamps used for both the imprints had a periodicity of $\sim 1.5 \mu \mathrm{m}$. Imprinting with the first stamp resulted in the formation of a perfect negative replica on the film surface, which were in the form of $750 \mathrm{~nm}$ stripes, as marked in the figure. The second imprint resulted in the holes along the raised channels created during the first imprinting. In figure 9(c) also, the first imprinting was done with a PVA foil with a periodicity of $1.5 \mu \mathrm{m}$, with stripe height $\sim 130 \mathrm{~nm}$, as shown in the figure. The second imprint, at a direction perpendicular to that of the first imprint was done with a patterned PVA foil having a periodicity of $\sim 800 \mathrm{~nm}$ and stripe height of $\sim 70 \mathrm{~nm}$, resulting in the array of rectangular pillars. By using different molds for different imprinting and varying the exposure time, host of ordered 2-D structures can be created, both on films coated on flat as well as curved substrates. In addition, there was no evidence of destruction or distortion of the imprinted patterns as well as no residue of the PVA stamp on the patterned films, when viewed under optical and AFM microscopes. Thus, by using the proposed technique, it is possible to obtain distortion free, large area patterns, which are perfect negative replicas of the original stamp on polymer films coated on curved substrates.

It is worth mentioning here that scanning of the patterns on the curved surfaces using an AFM was a slightly difficult issue. The model of the atomic force microscope available with the authors (Pico Plus; Molecular Imaging, USA) was particularly useful in this regard. In this particular model, all the three piezo electric sensors are mounted on the scanner, and the sample plate is free from all electronics. This feature was of particular help, as a specially designed sample plate with a large opening in the middle was used and the curved substrates were attached at the bottom of the sample plate. The patterned film surface was accessed by the cantilevers through the opening in the sample plate. Using this technique surface of tubes with diameters as large as $\sim 2 \mathrm{~cm}$ was successfully scanned.

3.3c Use of data containing CD foils as stamps: In the last part of our discussion, we focus our attention on one additional advantage that we could utilize for patterning polymer films, by all the methods described above, using patterned foils of optical data storage discs. That is, by pre-storing data on these discs, complex patterns could be created. In some of our experiments, instead of using foils from blank CDs and DVDs, we used the foil portion of CDs in which some data is written, that is some specially created data files were saved and written on them. Storage of data on optical disks resulted in more complex patterns on the foils, which in turn were used for subsequent patterning of the films. For example, writing of any data (in the form of a file) on an optical disk by a 'write laser' etches pits along the tracks on the polycarbonate 

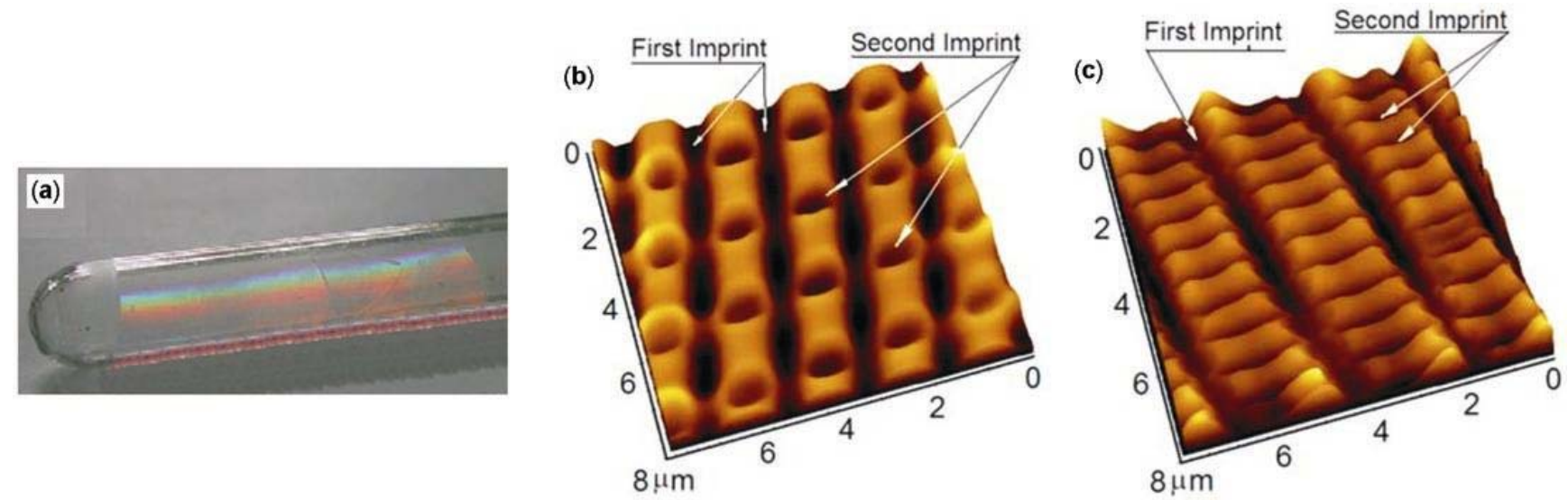

Figure 9. (a) Digicam image showing large area pattern on a PS film, coated on a cylindrical glass surface. The length of the patterned area is $\sim 40 \mathrm{~mm}$. (b and c) AFM images of patterns obtained on thin PS films coated on cylindrical glass substrate by solvent vapour assisted imprinting at room temperature, using flexible patterned PVA foils as stamps. (a) A 7.2 $\mu \mathrm{m}$ thick PS film imprinted twice with PVA foils having $1.5 \mu \mathrm{m}$ periodicity. The second imprint resulted in the holes along the raised channels created during the first imprinting. (b) A $4.8 \mu \mathrm{m}$ thick multiple imprinted PS film. The first imprint was done with a PVA stamp with $\sim 1.5 \mu \mathrm{m}$ periodicity and the second imprint with a $800 \mathrm{~nm}$ periodicity PVA foil, at right angles to the direction of the first imprint.

(a)

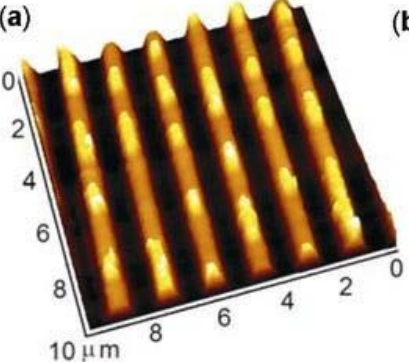

(b)

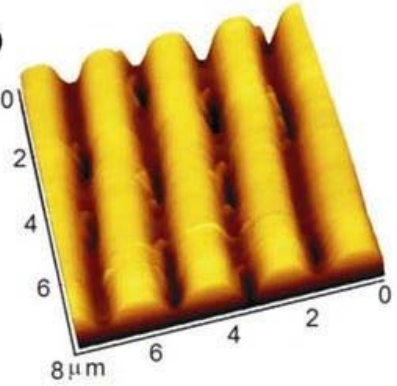

Figure 10. (a) AFM image of a patterned foil of a data containing $\mathrm{CD}$ with an array of raised pillars along the tracks and (b) a $850 \mathrm{~nm}$ thick PDMS film, imprinted with a data containing $\mathrm{CD}$ foil, resulting in structures having array of pits along the channels.

disk and matching 'burn marks' in the form of raised pillars on the adjoining metal foil. Briefly, it may be noted that the data is saved on the disc in a binary format; the flat portions of the etched track represent ' 0 ' while any change of the height along the track represents a binary ' 1 '. This laser burning process, clearly visible under the field of view of an atomic force microscope, results in an array of raised pillars ( $\sim 40 \mathrm{~nm}$ high and 1-2 $\mu \mathrm{m}$ long) along the tracks on the metal foil (figure 10(a)) of a CD. Storage of data onto a disc can, therefore, be used as a simple and inexpensive method for the creation of complex master pattern (in contrast to simple stripe patterned foils available from a blank disc), which can be subsequently used for patterning of soft surfaces following the methods already discussed. Storage of data on DVD does not create a topographic pattern (observed under AFM scans), as it alters the refractive index of an optically active coating layer.

For these particular experiments shown in figure 10, a text file of $\sim 700 \mathrm{MB}$ was created which contained only one alphabet. The size of the file was made $\sim 700 \mathrm{MB}$, as that matches the capacity of a CD and that the file physically spans across the entire disc i.e. the laser burning creates burn marks on the $\mathrm{CD}$. The present commercially available algorithm of data burning on a $\mathrm{CD}$ using a commercial CD writer, which is based on eight to fourteen modulation (EFM) algorithm does not allow a very clear one-to-one correspondence between any particular alphabet and its digital map. What it means is that any particular alphabet does not have a unique physical burn mark on the disc and there was virtually no difference in the AFM scans of the foils of two CDs, one having a file exclusively containing ' $\mathrm{P}$ ' and the other some other alphabet, say ' $\mathrm{B}$ '. Both of the foils showed raised burn marks along the raised stripes, as typically shown in figure 10 (a). We did test this by creating different data files with different alphabets exclusively, and also combination of alphabets, none of which showed any exclusive mapping pattern.

In spite of lack of short range order, by using foils of data written $\mathrm{CD}$, we could create patterns of polymer films, comprising of arrays of pits or holes along the floors of the tracks, as shown in figure 10(b). The particular example shown in this figure (10(b)) is for a $850 \mathrm{~nm}$ thick cross-linked PDMS film, imprinted with a data containing CD foil. The resulting structure consists of array of tiny pits (1-2 $\mu \mathrm{m}$ long) along the channels. These types of imbedded structures are otherwise difficult to make and could be useful in confined chemistry applications such as particle sorting, single molecule enzymology, growth of platelet crystals and wires and micro-fluidics. Thus, foils stripped from CDs, blank or data containing, indeed can be used as a low cost source of patterned foils 
that can be subsequently used for patterning of soft films and surfaces following the method presented.

\section{Conclusions}

In summary, by using inexpensive CD and DVD metal foils, which can also be pre-patterned by data writing, or any other patterned flexible foils (like PVA foils), we demonstrate very simple, room temperature, pressure-less, benchtop method for a rapid sub-micron and large area patterning of different types of polymer films like thermoplastics, soft elastic films and soft gels.

The paper presents two different strategies. While adhesion assisted room temperature imprinting, in which patterning is performed directly in the solid state by elastic deformations of the surface and its conformal contact with the patterned foil due to adhesive force is employed for patterning soft elastic films and soft gels, solvent vapour assisted imprinting has been reported for thermoplastic type polymers, in which the polymer film is softened by exposure to solvent vapour and subsequent patterning is achieved by deformation in the liquid state. Unlike the replica molding, there is neither mechanical compression of the mold, nor any liquid to solid phase transition induced shrinkage and pattern distortion. Use of a flexible foil, rather than a rigid stamp, is also a key ingredient of the proposed techniques. The foil stamps not only ensure a uniform conformal adhesive contact over large areas thus eliminating the need for a precise control of parallelism and application of uniform pressure required in the conventional imprint lithographies, but are also easy to peel, without resulting in any damage to the created patterns during stamp removal. In order to overcome the difficulty of stamp peel off and possible stamp rupture, water soluble PVA stamps have been used for patterning films by solvent vapour assisted imprinting. Additionally, patterning is done under gentle conditions, without application of excessive pressure, temperature and harsh chemical treatments. As patterning of hydrogel films does not even include the UV irradiation step, the method is especially suitable for applications involving structured hydrogels with embedded biomaterials and cells in tissue engineering, which can otherwise degrade under other processing conditions.

In general, any patterned foils, metallic or polymeric, including the ones produced with fast bulk methods such as Gravure printing, embossing etc are suitable for use as stamp in this technique. However, the use of readily available CD and DVD foils can already produce feature sizes as small as $800 \mathrm{~nm}$ and $400 \mathrm{~nm}$, respectively over large areas $\left(\sim \mathrm{cm}^{2}\right)$ because of the pattern splitting, which is another novel aspect of the technique. Finally, we also demonstrate that complex 2-D structures can be produced from 1-D stamps by partial hardening, rotation and reapplication of the stamp and patterning of films coated on non-planar curved surfaces. The simple technique proposed here is suitable for fabrication of low cost, large area templates for various bulk-nano applications. This 'do-it-yourself' method requires no special lithography facilities and can be particularly useful to the researchers routinely requiring patterned surfaces as templates, stamps and molds for rapid prototyping and experiments, for example, on optical and adhesive coatings, confined chemistry phenomena and biological applications.

\section{Acknowledgements}

This work was supported by DST Unit on Nanosciences, IIT Kanpur. One of the authors (AS) acknowledges the support of J.C. Bose Fellowship of the DST. (RM) acknowledges the financial support from DST fast track project.

\section{References}

Ansari K, van Kan J A, Bettiol A A and Watt F 2004 Appl. Phys. Lett. 85476

Bailey T, Choi B, Colburn M, Meissl M, Shaya S, Ekerdt J G, Sreenivasan S V and Willson C G $2000 \mathrm{~J}$. Vac. Sci. Technol. B18 3572

Beebe D J, Moore J, Bauer J M, Yu Q, Liu R H, Devadoss C and Jo B 2000 Nature 404588

Brabec C J, Sariciftci N S and Hummelen J C 2001 Adv. Funct. Mater. 1115

Bratton D, Yang D, Dai J and Ober C K 2006 Polym. Adv. Technol. 1794

Burn P L et al 1993 J. Am. Chem. Soc. 11510117

Burroughes J H, Bradley D D C, Brown A R, Mackay K, Friend R H, Burns P L and Holmes A B 1990 Nature 347539

Chin V I, Taupin P, Sanga S, Scheel J, Gage F H and Bhatia S N 2004 Biotech. Bioengg. 88399

Chou S Y, Krauss P R and Renstrom P J 1996 Science 27285

Chou S Y, Zhuang L and Guo L 1999 Appl. Phys. Lett. 751004

Chow A W 2002 AIChE J. 481590

Chowdhury D, Paul A and Chattopadhyay A 2001 Nano Letts 1 409

Deegan R D, Bakajin O, Dupont T F, Huber G, Nagel S and Witten T A 1997 Nature 389827

Di Bendetto F, Biasco A, Pisignano D and Cingolani R 2005 Nanotechnology 16 S165

Eichenbaum G M, Kiser P F, Simon S A and Needham D 1998 Macromolecules 315084

Emmelius M, Pawlowski G and Vollmann H W 1989 Angew. Chem. 1011475

Fischer P B and Chou S Y 1993 Appl. Phys. Lett. 622989

Gates B D, Xu Q, Stewart M, Ryan D, Willson C G and Whitesides G M 2005 Chem. Rev. 1051171

Ghatak A, Chaudhury M K, Shenoy V and Sharma A 2000 Phys. Rev. Lett. 854329

Ghatak A, Mahadevan L, Chung J Y, Chaudhury M K and Shenoy V 2004 Proc. R. Soc. London A460 2725

Gonuguntla M, Sharma A, Mukherjee R and Subramanian S A 2006a Langmuir 227066

Gonuguntla M, Sharma A and Subramanian S A 2006b Macromolecules 393365

Guo L J, Cheng X and Chou C-F 2004 Nano Letts 469

Harkema S, Schäffer E, Morariu M D and Steiner U 2003 Langmuir 199714 
He H, Cao X and Lee L J 2004 J. Controlled Release 95391

Henzie J, Barton J E, Stender C L and Odom T W 2006 Acc. Chem. Res. 39249

Hu Z, Zhang X and Li Y 1995 Science 269525

Hu Z, Chen Y, Wang C, Zheng Y and Li Y 1998 Nature 393 149

Ito Y 1999 Biomaterials 202333

Jacobs H O, Tao A R, Schwartz A, Gracias D H and Whitesides G M 2002 Science 296323

Jagur-Grodzinski J 2006 Polym. Adv. Technol. 17395

Kane R S, Takayama S, Ostuni E, Ingber D E and Whitesides G M 1999 Biomaterials 202363

Khang D -Y, Yoon H and Lee H H 2001 Adv. Mater. 13749

Khang D -Y, Kang H, Kim T -I and Lee H H 2004 Nano Lett. 4 633

Kim E, Xia Y and Whitesides G M 1995 Nature 376581

Kros A, Nolte R J M and Sommerdijk N A J M $2002 A d v$. Mater. 141779

Kumar A and Whitesides G M 1993 Appl. Phys. Lett. 632002

Lee K -B, Park S -J, Mirkin C A, Smith J C and Mrksich M 2002 Science 2951702

Lee T-W, Zaumsiel J, Kim S H and Hsu J W P 2004 Adv. Mater. 162040

Lei M, Gu Y, Baldi A, Siegel R A and Ziaie B 2004 Langmuir 208947

Levene M J, Korlach J, Turner S W, Foquet M, Craighead H G and Webb W W 2003 Science 299682

Lopez W A and Jaeger H M 2001 Nature 414735

Lupton J M, Koeppe R, Muller J G, Feldmann J, Scherf U and Lemmer U 2003 Adv. Mater. 151471

McDonalds J C, Duffy D C, Anderson J R, Chiu D T, Wu H, Schueller O J A and Whitesides G M 2000 Electrophoresis 2127

McFarland C D, Thomas C H, DeFilippis C, Steele J G and Healy K E 2000 J. Biomed. Mater. Res. 49200

Meyer J -U 2002 Sensors and Actuators A97-98 1

Mukherjee R, Gonuguntla M and Sharma A 2007a J. Nanosci. Nanotechnol. 72069

Mukherjee R, Pangule R C, Sharma A and Banerjee I 2007b J. Chem. Phys. 127064703
Ouyang M, Muisener R J, Boulares A and Koberstein J T 2000 J. Membrane Sci. 177177

Reiter G 1992 Phys. Rev. Lett. 6875

Reiter G 1993 Langmuir 91344

Rhee S W, Taylor A M, Tu C H, Cribbs D H, Cotman C W and Jeon N L 2005 Lab on a Chip 5102

Rondelez Y, Tresset G, Tobata K V, Arata H, Fujita H, Takeuchi S and Noji H 2005 Nature Biotechnol. 23361

Ruchhoeft P et al 1999 J. Vac. Sci. Technol. B17 2965

Salleo A, Wong W S, Chabinyc M L, Paul K E and Street R A 2005 Adv. Funct. Mater. 151105

Schäffer E, Thurn-Albrecht T, Russell T P and Steiner U 2000 Nature $\mathbf{4 0 3} 874$

Schaper C D 2003 Nano Lett. 31305

Schift H, Heyderman L J, Auf der Maur M and Gobrecht J 2001 Nanotechnology 12173

Sharma A 2003 Eur. Phys. J E 12397

Sharma A and Reiter G 1996 J. Colloid Interface Sci. 178383

Sharma A and Khanna R 1998 Phys. Rev. Lett. 813463

Sharma A and Reiter G 2002 Phase Trans. 75377

Singh A, Jayaram J, Madou M and Akbar S 2002 J. Electrochem. Soc. 149 E78

Stellacci F 2006 Adv. Funct. Mater. 1615

Suh K Y, Kim Y S and Lee H H 2001 Adv. Mater. 131386

Tan H, Gilbertson A and Chou S Y 1998 J. Vac. Sci. Technol. B16 3926

Tanaka T, Fillmore D, Sun S T, Nishio I, Swislow G and Shah A 1980 Phys. Rev. Lett. 451636

Terfort A, Bowden N and Whitesides G M 1997 Nature 386 162

Unger M A, Chou H, Thorsen T, Scherer A and Quake S R 2000 Science 288113

Whitesides G M 2003 Nature Biotechnol. 211161

Whitesides G M and Grzybowski B 2002 Science 2952418

Whitesides G M, Mathias J P and Seto C T 1991 Science 254 1312

Widawski G, Rawiso M and Francois B 1994 Nature 369387

Xia Y, Kim E, Zhao X -M, Rogers J A, Prentiss M and Whitesides G M 1996 Science 273347

Xia Y and Whitesides G M 1998 Angew. Chem. Int. Ed. 37550 